\title{
Utilisation de T. evansi \\ pour la recherche des hydrolases sanguines responsables de la formation de glucose à partir de différents diholosides et du glycogène
}

\begin{abstract}
par J. BALIS - avec la collaboration technique de Madame CHATELAIN.
\end{abstract}
\begin{abstract}
RÉSUMÉ
L'auteur expose le principe el la réalısation technique d'une méthode origınale, utilisant $T$. evansi comme réactif, et permetiant la recherche de certaines hydrolases sanguines.

Par cette méthode, ont été mises en évidence une maltase dans le sang du cheval, du bœuf, du lapin et du rat, ef une glycogénase dans le sang du bøuf. du lapin et du rat.
\end{abstract}

Lors d'un précédent travail (1) consacré au métabolisme glucidıque de $T$, evansı nous avions trouvé que ce flagellé était apparemment capable d'utiliser outre 3 hexoses (glucose, fructose, mannose), le maltose et le glycogène.

En réalité ces deux derniers corps, pour être métabolisés doıvent au préalable subir l'action d'une hydralase sanguine, les transformant en glucose directement assimilable par T. evansi (3).

L'objeł du présent traval est de décrire une technique originale utilisant $T$, evansi comme réaltif et permettant de mettre en évidence ces hyedralases dans le sang de différentes espèces animales.

\section{Matériel et méthodes}

T. evansı ne métabolise que 3 glucides (glucase, fructose et mannose) et consomme de l'oxygène en présence de ces sucres.

Ce fait peut-être mis en évidence à l'aide d'une suspension d'hématies dont la couleur passe très nettement du rouge clair au rouge vineux dès que l'hérnoglobıne est réduite.

Le virage se produit en 30 à 60 minutes si le rombre de trypanosomes est important. Lors- qu'on introduit dans le milieu un diholoside susceptible d'être hydrolysé par une diastase sanguine, le changement de teinte apparaît beaucoup plus tardivement en raison du temps nécessarre à la formation de glucose en quantité suffisante.

Il est donc possible de déceler une hydrolase sanguine en comparant les résultats obtenus dans les tubes suivants :

No 1.

Dilution de sang à analyser $\frac{1}{i}$ solution de diholoside.

+24 heures après, dilution de sang de rat fortement parasité.

No 2. - (effectué en même temps, que la 2e partie du no 1).

Dilution de sang à analyser + solution de diholoside + dilution de sang de rat fortement parasifé.

$S_{1}$ le diholaside est hydrolysé par une diastase sanguine, on constate un virage dans le tube $n^{0} 1$ alors que la teinte du tube $n^{\circ} 2$ ne varie pas.

La réalısation pratique de la méthode est conforme au tableau no 1. 
On utilise 6 séries de 6 tubes stérilisés qu four Pasteur et numérotés de 1 à 36 . Le milieu de dilution a la composition suivante :

- Phosphate bipotassique ..... $1 \mathrm{~g}$

- Chlorure de sodium ........ $0,25 \mathrm{~g}$

- Eau distillée............. $100 \mathrm{ml}$

- Phosphate monopotassique'. Q S pour obtenir $\mathrm{pH} 7,4$

- Stérilisation par chauffage ou filtration sur Seitz.

Les sucres sont utilisés sous forme de solutions à 1 p. 1.000 stérilises par filtration.

Ce procédé convient mal au glycogène qui est partiellement retenu sur le disque d'amiante. De ce fait on n'obtient plus exactement une solution à 1 p. 1.000 ef pour ce corps les résultats ne peuvent être que qualitatifs.

Le sang de rat parasité ainsı que le sang à analyser sont dilués stérilement au 1/30 dans le milıu précédemment décrit. Les séries $1,2,3$ et 5 sont préparées aseptiquement.

Après 24 heures on' réalise les séries 4 et 6 puis on répartit rapidement la dilution de sang de rat parasité dans les tubes 13 à 36 .

Le nombre de trypanosomes dans le sang de rat doit au mons atteindre 500.000 par millimètre cube si on veut avoir un virage très net.

En examinant le tableau na 1 on voit que la réaction est effectuée sur des concentrations croissantes de sucre, car les vitesses d'hydrolyse et d'utilisation du glucose formé sont proportionnelles à la concentration du substrat.

Après quelques essais nous avons adopté les dilutions suivantes:

$1 / 45.000-1 / 22.500-1 / 15.000-1 / 11.250$ $-1 / 9.000$ auxquellès on ajoute un tube témoin, ne contenant pas de sucre (tubes nos $1,7,13,19$, $25,31)$.

Les séries 1 et 2 permettent de vérifier qu'il n'y a pas de virage en l'absence de T. evansi.

La comparaison des séries 5 et 6 décèle la présence de l'hydrolase recherchée ef par rapport à la série no 3 (contenant du glucose), on peut évaluer son activité.

Enfin l'examen comparatif des séries 3 et 4 permet d'apprécier l'importance de la glycolyse sanguine en 24 heures.'

Pour fixer les idées nous donnerons un exemple concret :
La recherche de la maltase dans le sang de rat fournit les résultats suivants :

Série $n^{\circ}$

$\begin{array}{lllllll}\text { I, } & 0 & 0 & 0 & 0 & 0 & 0 \\ 2, & 0 & 0 & 0 & 0 & 0 & 0 \\ 3 . & 0 & 0 & 1 & 4 & 4 & 4 \\ 4, & 0 & 1 & 4 & 4 & 4 & 4 \\ 5, & 0 . & 0 & 0 & 3 & 4 & 4 \\ 6, & 0 & 0 & 0 & 0 & 0 & 0\end{array}$

Le chiffre 4 représente le virage maximum. Ce dernier est obligatoirement obtenu dans le tube n० 24 de la série 4.

\section{Avantages de la méthode}

Elle ne nécessite qu'un matériel réduit et permèt de déceler qualitativement une hydrolase et doser son activité.

II est également possible d'évaluer l'importance de la glycolyse sanguine.

\section{Inconvénients}

Cette méthode nécessite un sang de rat très fortement parasité. Elle est valable chaque fois que le résultat est positif.

Si l'hydrolase recherchée est dovée d'une très fable activité, il est alors impossible d'obtenir un virage. Dans ce cas, et si on tient compte des hydrolases. susceptibles d'exister dans le sang de rat, on augmente considérablement la sensi bilıté en comparant la mobilité des trypanosomes (1) dans le premier et le dernier tube des séries 5 et 6 . En effet une très faible quantité de glucose ou de fructose est capable de provoquer une mobilisation et, en l'absence de cette dernière, on peut affirmer que si l'hydrolase existe, son activité est négligeable dans les conditions de l'expérience, c'est-à-dire diluée au $1 / 30$ et à la température de $25^{\circ}$.

\section{Résultats et discussion}

A l'aide de cette technique nous avons recherché la présence de 5 diastases (lactase, maltase, saccharase, tréhalase, glycogénase), dans le sang des 6 espèces suivantes :

Homme, cheval, bœuf, lapin, rat, cobaye.

Les résultats consignés dans le tableau $n^{\circ} 2$ doivent être considérés comme une illustration de la méthode car la plupart d'entre eux sont le 
TABLFAD HO I

\begin{tabular}{|c|c|c|c|c|c|c|}
\hline \multicolumn{7}{|c|}{ Série No I } \\
\hline Tube $n^{0}$ & $I$ & 2 & 3 & 4 & 5 & 6 \\
\hline Sg. dillué (en ml) & 4 & 4 & 4 & 4 & 4 & 4 \\
\hline Glucose (en gouttes) & & 2 & 4 & 6 & 8 & 10 \\
\hline Eau physiologique (en goutteo) & 10 & 8 & 6 & 4 & 2 & \\
\hline \multicolumn{7}{|c|}{ Série $\mathbb{N}^{0} 2$} \\
\hline Tube $n^{0}$ & 7 & 8 & 9 & 10 & 11 & 12 \\
\hline Sg. dilué (en mll) & 4 & 4 & 4 & 4 & 4 & 4 \\
\hline Sucre ́́tudié (en gouttes) & & 2 & 4 & 6 & 8 & 10 \\
\hline Eau physzologzque & 10 & 8 & 6 & 4 & 2 & \\
\hline \multicolumn{7}{|c|}{ Série No 3} \\
\hline Tube $n^{0}$ & 13 & 14 & 15 & 16 & 17 & 18 \\
\hline Sg did.ué (en ml) & 2 & 2 & 2 & 2 & 2 & 2 \\
\hline Glucose (en trouttes) & & 2 & 4 & 6 & 8 & 10 \\
\hline Bau physiologique & 10 & 8 & 6 & 4 & 2 & \\
\hline Sp, dilué (on ml) & 2 & 2 & 2 & 2 & 2 & 2 \\
\hline \multicolumn{7}{|c|}{ Série $\mathrm{H}^{0} 4$} \\
\hline Tube No & 19 & 20 & 21 & 22 & 23 & 24 \\
\hline Sg. dilué (en mI) & 2 & 2 & 2 & 2 & 2 & 2 \\
\hline Glucose (en goutles) & & 2 & 4 & 6 & 8 & 10 \\
\hline Eau physiologinue (en gouttes) & 10 & 8 & 6 & 4 & 2 & \\
\hline Sp. dilué (en ml) & 2 & 2 & 2 & 2 & 2 & 2 \\
\hline \multicolumn{7}{|c|}{ Série झ̊ 5} \\
\hline Tube 150 & 25 & 26 & 27 & 28 & 29 & 30 \\
\hline Sg. dilué (en ml) & 2 & 2 & 2 & 2 & 2 & 2 \\
\hline Sucre étudié (en gouttes) & & 2 & 4 & 6 & 8 & 10 \\
\hline Eau physiolozique (en gruttes) & 10 & 8 & 6 & 4 & 2 & \\
\hline Sp. dilué (en ml) & 2 & 2 & 2 & 2 & 2 & 2 \\
\hline \multicolumn{7}{|c|}{ Bérie $\mathbb{N}^{\circ} 6$} \\
\hline Tube $\mathbb{N}^{\circ}$ & 31 & 32 & 33 & 34 & 35 & 36 \\
\hline Sg. dilué (en ml) & $2^{\prime}$ & 2 & 2 & 2 & 2 & 2 \\
\hline Sucre étudié (en gouttes) & & 2 & 4 & 6 & $B$ & 10 \\
\hline Eau physiologique (en gouttes) & 10 & 8 & 6 & 4 & 2 & \\
\hline Sp. dilué (en ml) & 2 & 2 & 2 & 2 & 2 & 2 \\
\hline
\end{tabular}

$\mathrm{Sg}=\operatorname{sang}$ dans lequel on recherche Ia diastase.

Sp.= sang de rat fortement parasité. 


\begin{tabular}{|c|c|c|c|c|c|c|c|c|c|c|c|c|}
\hline & \multicolumn{2}{|c|}{ Lactase } & \multicolumn{2}{|c|}{ MaItase } & \multicolumn{2}{|c|}{ Seccherase } & \multicolumn{2}{|c|}{ Tréhalase } & \multicolumn{2}{|c|}{ Glycogènase } & \multirow[b]{2}{*}{ Série 3} & \multirow[b]{2}{*}{ Sérile 4} \\
\hline & Virage & $\begin{array}{l}\text { Test } \\
\text { mobi- } \\
\text { lité }\end{array}$ & Virege & $\begin{array}{l}\text { Teat } \\
\text { mobi- } \\
\text { Ilté }\end{array}$ & Virage & $\begin{array}{l}\text { Test } \\
\text { mobi- } \\
\text { lité }\end{array}$ & Virage & $\begin{array}{l}\text { Test } \\
\text { mobj- } \\
\text { lité }\end{array}$ & Virage & $\begin{array}{l}\text { Test } \\
\text { mobi- } \\
\text { lité }\end{array}$ & & \\
\hline Homne & 0 & - & 0 & - & 0 & - & 0 & - & 0 & - & 1444 & 3444 \\
\hline Cheval & 0 & - & 1444 & + & 0 & - & 0 & - & 0 & - & 1444 & 2444 \\
\hline Boeuf & 0 & - & 2444 & + & 0 & - & 0 & - & $244^{\prime}$ & + & 2444 & 3444 \\
\hline Lapin & 0 & - & 3 & + & 0 & - & 0 & - & 2 & + & 1444 & 2444 \\
\hline Rat & 0 & - & 344 & + & 0 & - & 0 & - & 12 & + & 1444 & 14444 \\
\hline Cobaye & 0 & - & 0 & - & 0 & - & 0 & - & 0 & - & 2444 & 3444 \\
\hline
\end{tabular}

fruit d'une expérience unique et ne peuvent donc être généralisés.

La diastase la plus fréquente est la maltase qu'on retrouve chez 4 espèces animales.

La glycogènase est plus rare et surtouł moins active.

Nous n'avons pu mettre en évidence la tréhalase signalée par BOWMAN, VON BRAND et TOBIE (2) dans le sang de l'homme et de lapin. Cette diastase avait été invoquée par ces auteurs pour expliquer les résultats obtenus par Weinman avec le tréhalose sur $T$. rhodesiense ef $T$. gambiense (4).

L'examen des séries 3 ef 4 a permis de noter régulièrement une glycolyse appréciable en 24 heures, ceci se manifestant toujours par un virage moins important dans la série 3. Par contre il est nettement plus précoce dans cette même série, comme sı le glucose avait subı une sorte de préparation, le rendant plus apte d̀ être utılisé par $T$. evansi.

\section{Conclusions}

Nous avons exposé dans ce travail, le principe et la réalisation technique d'une méthode originale permetiant la recherche de certaines hydrolases dans les liquides biologiques.

Elle est basée sur le fait que $T$. evansi consomme de l'oxygène et se mobilise, uniquement en présence de glucose, fructose et mannose.

Un diholoside ne peut donc provoquer ces phénomènes que s'ıl est préalablement hydroiysé et transformé en l'un de ces 3 hexoses.

Cette méthode nous' a permis de mettre en évidence, dans le sang de différentes espèces animales, une maltase '(cheval, bœuf, lopin, rat) et une glycogènase (bœuf, lapin, rat).

\section{SUMMARY}

\section{Use of $T$. evansi for the detection of hydrolases of blood which are responsible of dextrose formation from various di-saccharides and glycogen}

The author has reported the principle and the practical application of an inventive method using $T$. evansi as a reagent which. allows the detection of various hydrolases of blood.

With this method a maltase has been shown in horse, ox, rabbit and ra blood, a glycogenase in ox, rabbit and rat blood. 


\section{RESUMEN}

Utilizaciôn de T. evansi para la busqueda de las hidrolasis sanguíneas, responsables de la formaciôn de glucosa a partir de diferentes diholosidos y del glicógeno

El autor expone el principio y la realizaciôn tecnica de un método original, utilizando $T$, evansi como reactivo, y permitiendo la busqueda de ciertas hidrolosis sanguíneas.

Mediante este método, fueron descubiertas una maltasa en la sangre de un caballo, de un buey, de un conejo y de una rata, y una glicogenasis en la sangre de un buey, de un conejo y de una rata.

\section{BIBLIOGRAPHIE}

1. J. BALIS. - Utilisation des glucides ef de leurs produits de métabolisme par $T$. evansi et T: brucei. Sous-presse.

2. I. B. R. BOWMAN, T. VON BRAND et E.J. TOBIE. - The cultivation and metabolism of trypanosomes in the presence of trehalose with observations on trehalase in blood serum. Exp. Parásit. 1960, 10 274-283.

3. P. B. MARSHALL. - The glucose metabolism: of $T$. evansi and the action of trypanocides. Brit. j. Pharmacoil. 1948, 3, (8), 8-14.

4. D. WEINMAN. - Trehalose metabolism of Tryponosomes. Nature, 1960, 186, 166. 